Repository of the Max Delbrück Center for Molecular Medicine (MDC) Berlin (Germany)

http://edoc.mdc-berlin.de/11714/

\title{
Development and evaluation of a small and mobile Magneto Alert Sensor (MALSE) to support safety requirements for magnetic resonance imaging
}

Conrad Martin, Tobias Frauenrath, Celal Özerdem, Wolfgang Renz, and Thoralf Niendorf 
Development and Evaluation of a Small and Mobile Magneto Alert Sensor (MALSE) to Support Safety Requirements for Magnetic Resonance Imaging

Conrad Martin $^{1}$, Tobias Frauenrath ${ }^{1}$, Celal Özerdem ${ }^{1}$, Wolfgang Renz ${ }^{1,2}$, Thoralf Niendorf ${ }^{1,3}$

${ }^{1)}$ Berlin Ultrahigh Field Facility (B.U.F.F.), Max-Delbrueck Center for Molecular Medicine, Berlin, Germany

2) Siemens Healthcare, Erlangen, Germany

3) Experimental and Clinical Research Center (ECRC), Charité Campus Buch, Humboldt-University, Berlin, Germany

Address correspondence to: Prof. Dr. Thoralf Niendorf, Director, Berlin Ultrahigh Field Facility (B.U.F.F.) Max-Delbrueck Center for Molecular Medicine Robert-Roessle-Strasse 10 13125 Berlin

Germany

phone: +490309604 4504

fax: $\quad+49030960449178$

e-mail: thoralf.niendorf@mdc-berlin.de 


\section{$\underline{\text { Abstract }}$}

Objective: The purpose of this study is to (i) design a small and mobile Magnetic field ALert SEnsor (MALSE), (ii) to carefully evaluate its sensors to their consistency of activation/deactivation and sensitivity to magnetic fields, and (iii) to demonstrate the applicability of MALSE in 1.5 T, 3.0 T and 7.0 T MR fringe field environments.

Methods: MALSE comprises a set of reed sensors, which activate in response to their exposure to a magnetic field. The activation/deactivation of reed sensors was examined by moving them in/out of the fringe field generated by 7TMR.

Results: The consistency with which individual reed sensors would activate at the same field strength was found to be $100 \%$ for the setup used. All of the reed switches investigated required a substantial drop in ambient magnetic field strength before they deactivated.

Conclusions: MALSE is a simple concept for alerting MRI staff to a ferromagnetic object being brought into fringe magnetic fields which exceeds MALSEs activation magnetic field. MALSE can easily be attached to ferromagnetic objects within the vicinity of a scanner, thus creating a barrier for hazardous situations induced by ferromagnetic parts which should not enter the vicinity of a MR-system to occur.

Key Words: MRI, MR safety, magneto alert sensor, high field MRI, ultrahigh field MRI 


\section{$\underline{\text { Introduction }}$}

Magnetic forces of fringe magnetic fields of MR systems on ferromagnetic components can impose a severe patient, occupational health and safety hazard. MRI accidents are listed as number 9 of the top 10 risks in modern medicine [1-2]. With the advent of (ultra)high field MR systems [3-14] this risk, which is commonly known as the missile or projectile effect is even more pronounced. These projectiles usually consist of common office and hospital items that contain a fair amount of ferromagnetic metal including for example hospital beds, intra venous poles, oxygen tanks, and conventional ECG devices used for patient monitoring, computer displays, ventilator etc. [15]. Most MRI accidents occur when non-MRI personnel (or careless MRI workers) introduce ferromagnetic objects into the magnetic environment. It is estimated that the reported incidents only account for about $10 \%$ of the actual number of such incidents, and even in this case, the number of incidents has jumped approximately 300\% from 2004 to 2008 [16-17], ranging from mechanical damage to patient death [15,18]. There have been at least 33 such accidents reported in the last 5 years [18-19], as well as at least 4 reported deaths over the last 10 years [19]. These casualties are probably most widely known through television documentaries and printed media [20-22] but still present the tip of the iceberg of MR safety violations.

Various policies [23-26] have been implemented to safeguard healthcare workers, volunteers and patients with the ultimate goal of avoiding unforeseen disasters and injuries due to ferromagnetic objects. These measures safety initiatives and awareness campaigns spearheaded by scientific organizations and other bodies and include safety training, risk reduction strategies, occupational health instructions, safety guidelines and warning signs. These safety procedures are commonly supplemented by metal or ferromagnetic detectors which are positioned at the entrance of the MR scanner room for example, as well as hall sensors [27] and other magnetic field sensing devices. The costs of traditional metal or 
ferromagnetic detectors are significant. Stand alone detector configurations and handheld scanners are frequently not properly used due to the heavy work load as well as busy environment experienced by hospital staff. Furthermore, some detectors used in current clinical practice do not distinguish between ferromagnetic and non ferromagnetic objects, thus making it difficult for hospital staff to maintain MR safety. Warning labels on the doors, walls or on the ground denoting the $5 \mathrm{G}$ and $10 \mathrm{G}$ are likely to be overlooked in a fast paced hospital environment. Thus auditory or visual warning of ferromagnetic objects being brought into the MR environment is necessary.

Recent designs have typically been laid out as strip line elements on rigid or semiflexible frames [7]. A strategy employing small magnetic field alert sensors which can be attached to ferromagnetic objects that are commonly used in a clinical environment is conceptually appealing for the pursuit of reducing the risk of ferromagnetic projectile accidents. Hence, the first aim of this study is to design a simple, cost-effective and mobile magneto alert sensor (MALSE) which provides alarm in the presence of static magnetic fields and which can be used in various configurations. Next we evaluate reed contacts which are activated by magnetic fields as to their consistency of activation/deactivation and sensitivity to magnetic fields, given that MALSE makes use of such components. Lastly we examine the applicability of MALSE in 1.5 T, 3.0 T and 7.0 T whole body MR fringe field environments. 


\section{Materials and Methods}

This study was approved by the local institutional ethics committee in order to be in full compliance with local requirements. Informed written consent was obtained from each subject (healthy volunteers, $n=10$ ) prior to the study.

MALSE comprises three main components as illustrated in Fig. 1:

Power supply: The current MALSE implementation is powered by a (e.g. lithium) battery. The device does not consume battery power if not activated. Consequently the battery power will not run out as the battery itself is not drained unless the MALSE sensor is active. Therefore the battery lifetime is only limited by the usual idle battery lifetime (up to 10 years for lithium batteries). The sensors should be exchanged before the batteries lifespan expires.

Signal unit: An acoustical (buzzer) signal unit is used to generate an alert. The use of piezo signal generators is preferred because of their small dimensions and slim geometry. Piezo devices also come with the benefit of being suitable for ultrahigh magnetic fields. Alternatively, optical (e.g. LED) signals can be used to generate an alert.

Magnetic field alert sensor: The sensor uses seven magnetic switches. These "reed contacts" are set on equal angles from each other as indicated in Fig. 1b. The reason for using seven is that a reed contact is most susceptible to being activated by a magnetic field when its long axis is aligned with magnetic field lines. By having the reed contacts placed in the orientations described above there can be no more than 27.4 degrees between any one reed contact and the magnetic field lines before the field lines move to a smaller angle than this to one of the other reed contacts. In this way it is ensured that the device will generate an alarm no matter in what orientation it is. 
The unit of magnetic strength used to describe reed contacts is Ampere-Turn (AT). The relationship between magnetic field strength, distance from the magnet at activation and the sensitivity of the reed switches is complex, and is determined by the size and shape of the magnet, as well as the shape and position of the reed contacts involved. Reed contacts are manufactured to within a certain range of AT values. Typically reed contacts cover broad ranges of AT values such as 10 to $30 \mathrm{AT}$ (Stock \#: 503800-62, Conrad Electronic SE Klaus-Conrad-Str.1 92240 Hirschau, Germany) and 5 to 15 AT (Stock \#: 118-7120, RS Components Ltd. Birchington Road, Corby, Northants, NN17 9RS, UK) used here. It is essential to examine whether or not each individual reed contact is consistently activated at a specific magnetic field strength when it is aligned with the magnetic field lines. Hence we scrutinized the consistency of activation and sensitivity to magnetic fields of individual reed contacts. For this purpose three sets of experiments were performed: First, it was measured that each reed contact would yield reproducible results in that it would close at a consistent field strength. Second, it was examined whether the reed contact is sensitive enough to be activated in magnetic fields $\left(\mathrm{B}_{0 \text { (act) }}\right)$ slightly greater than the $5 \mathrm{G}$ threshold. Finally, the activation/deactivation ( $\left.\Delta \mathrm{B}_{0 \text { (act-deact) }}\right)$ behavior was assessed to make sure that once a reed contact is closed it will have to be moved a significant distance away from the activation area to be re-opened, thus ensuring that MALSE must be taken outside of the activation zone before it deactivates.

The magnetic field strength was mapped along the main axis of a fringe field generated by a $7 \mathrm{~T}$ whole body MR system (Magnetom, Siemens, Erlangen, Germany) using a Sypris 5180 gauss meter. For each of the two cohorts of reed contact types used ( $n=35$ of the 10 to 30 AT type and $n=30$ of the 5 to 15 AT type) the reed contact activation/deactivation points were measured using a customized pulley system attached to an electric motor and controlled by a microcontroller The reed contact under test (RUT) was moved across the magnetic field lines 
until it was activated. This was recorded by a processing unit connected to the microcontroller using an RS232 interface. At this point, the direction of motion was inverted to record the deactivation point. For this purpose the motors' polarity and therefore the travel direction of RUT was also controlled by the processing unit. Subsequently, RUT was moved to the home position which was used as a reference. To examine the reproducibility and to exclude any hysteresis effect, each RUT was moved back and forth 5 times. 


\section{$\underline{\text { Results }}$}

The number of reed contacts that activated in a given $0.25 \mathrm{G}$ range is shown in Fig. 2. The portfolio of reed contacts included in this study showed activation at magnetic field strengths ranging from $7 \mathrm{G}$ to $16.5 \mathrm{G}$ as illustrated in Fig. 2. Reed contacts with a 5 to $15 \mathrm{AT}$ specification showed activation for magnetic fields strengths of 7 G to 16.5 G. Out of 30, only one activated in the range of $7 \mathrm{G}$. The 10 to 30 AT contacts' activation range was much more condensed. Reed contacts with a 10 to 30 AT specification got activated for magnetic field strengths ranging from 8 to $12 \mathrm{G}$. Even though the average field strength at activation of the 10 to $30 \mathrm{AT}$ contacts $\left(\Delta \mathrm{B}_{0 \text { (act) }}=9.5 \pm 1.1 \mathrm{G}\right)$ was slightly less than that of the 5 to $15 \mathrm{AT}$ contacts $\left(\Delta \mathrm{B}_{0 \text { (act) }}=10.8 \pm 3.1 \mathrm{G}\right)$, the 5 to $15 \mathrm{AT}$ contacts had 6 in the $7 \mathrm{G}$ to $8 \mathrm{G}$ range as well as 5 in the $8 \mathrm{G}$ to $9 \mathrm{G}$ range, whereas the 10 to 30 AT contacts had only 10 in the $8 \mathrm{G}$ to $9 \mathrm{G}$ range.

The activation/deactivation behavior of each reed contact is surveyed in Fig. 3. The deactivation magnetic field strengths of the reed contact, in conjunction with the activation magnetic field strengths were used to create a graph of the activation magnetic field strength vs. the difference between the activation and deactivation magnetic field strengths. The average change in magnetic field strength is greater for the 5 to 15 AT reed contacts $\left(\Delta \mathrm{B}_{0 \text { (act- }}\right.$ deact $)=5.7 \pm 3.2 \mathrm{G})$ than for the 10 to $30 \mathrm{AT}$ reed contacts $\left(\Delta \mathrm{B}_{0 \text { (act-deact) }}=1.3 \pm 0.8 \mathrm{G}\right)$. This means that any magnetic field alert sensor built with them will have to be taken at least this far away from the field that activates it before it will deactivate.

The consistency with which individual reed contacts would activate at the same field strength was tested by selecting 3 reed contacts from each type to be tested 35 times, and by testing all remaining reed contacts 5 times. The variance in activation field strength for all of these tests was too small to be relevant for the setup used, indicating that the consistency in the activation field strength of all the reed contacts is very high. Five of each category of reed 
contacts were also tested at 27.4 degrees with respect to the $\mathrm{B}_{0}$ vector. In all cases this resulted in the reed contact being 5 to $10 \%$ more sensitive to the magnetic field. This means that activation was reached at magnetic field strengths $5 \%$ to $10 \%$ smaller than that obtained for parallel alignment of the reed contact with the magnetic field so that MALSE will be more sensitive to an ambient magnetic field if it is moved into the ambient magnetic field in said orientation vs. a straight parallel alignment. Five of each type of reed contacts were tested 6 months after the initial experiments, during which time they had been present in the fringe field of a 7T MR system. The sensitivity of the 10 to 30 AT reed contacts changed by no more than $3.2 \%$. The sensitivity of the 5 to 15 AT reed contacts changed by no more than $1.9 \%$.For proof of concept a prototype made of standard electronic components was realized (Fig. 4). Of course, even more miniaturized versions are possible, in particular when using a reduced buzzer size. The proposed MALSE approach was examined in a clinical environment using a more sophisticated implementation (Fig. 4). For this purpose only selected reed contacts with activation at 7 to $8 \mathrm{G}$ only were included. MALSE's applicability and efficacy was tested in a clinical environment using the fringe field of our local 1.5 T, 3.0 T and 7.0 T whole body MR systems (Fig. 5). Fig. 5 demonstrates that MALSE was sensitive and powerful enough to generate a visual alert using the built-in LED for positions placed at the $10 \mathrm{G}$ iso-contour lines of the magnetic field. For the $1.5 \mathrm{~T}$ installation used, MALSE was activated inside of the scanner room in a location very close to the scanner rooms door (Fig. 5). For the $3.0 \mathrm{~T}$ installation used, MALSE was activated $1.5 \mathrm{~m}$ away from the front end of the patient table (Fig. 5). For the 7.0 $\mathrm{T}$ installation used MALSE was activated in the operator room (Fig. 5). 


\section{$\underline{\text { Discussion }}$}

The feasibility and efficacy of a magneto alert sensor MALSE which uses reed contacts to provide alarm in the presence of a given static magnetic field have been shown for $1.5 \mathrm{~T}, 3.0 \mathrm{~T}$ and 7.0 T MR systems. The assessment of 5 to $15 \mathrm{AT}$ and 10 to $30 \mathrm{AT}$ reed contacts demonstrated that it is quite possible to build magneto alert sensor using reed contacts commercially available. Of course, reed contacts would have to be screened for their effectiveness and only those that activate under $10 \mathrm{G}$ would be selected for use in a MALSE device. Although many of the 10 to 30 AT contacts have deactivation distances that are quite short, there is a large enough number of reed contacts in the sample which have relatively long deactivation distances. One would thus have to select reed contacts that provide long activation to deactivation distance and activate at a relatively low magnetic field strength for use in MALSE.

The magnetic field strength of $8 \mathrm{G}$, is well outside the scanner room of a $7.0 \mathrm{~T}$ scanner installation. Even for an actively shielded 3.0 T scanner, the $8 \mathrm{G}$ line is approximately 2-3 $\mathrm{m}$ from the magnets iso-center. In fact, a field strength of $8 \mathrm{G}$ is definitely far too weak to create a noticeable force on a ferromagnetic object in that area. It is thus quite possible to mass produce MALSE devices and attach them to every ferromagnetic object in the vicinity of an MRI scanner at a hospital or research facility using adhesives because MALSE is easy and cheap to build.

The design of MALSE can also be refined with the addition of magnetic or ferromagnetic components which would allow to increase as well as to precisely control the activation and deactivation field strengths. Also, an extension of the MALSE design can be anticipated to evolve towards a warning device which provides alerts for $\mathrm{dB} / \mathrm{dt}$ levels which exceed the thresholds defined by the IEC and other regulatory/governmental bodies. 
There are other devices which have been designed to help keep ferromagnetic equipment outside of the MRI exclusion zone including stand alone and mountable configurations. The costs of traditional stand alone ferromagnetic detectors are significant. Stand alone detector configurations are frequently not properly used due to a busy clinical environment. The physical size and weight of current mobile magnetic field strength alarm systems [28] render it unsuitable - if not prohibitive - to be mounted to mid size ferromagnetic objects such as notebooks or medical trays, let alone small size ferromagnetic objects such as scissors.

As the size of MRI rooms decrease and magnetic field strengths increase, it will become increasingly important to keep ferromagnetic objects in areas only where they do not exhibit any safety hazards. To this end, the MALSE sensor is a simple and effective concept for alerting MRI staff to a ferromagnetic object being brought into fringe magnetic fields which are larger than MALSEs activation magnetic field. This will help to prevent accidents due to the ferromagnetic missile effect if implemented correctly. It should be emphasized that MALSE devices are meant to provide a supplemental level of safety in the MR environment and are in no way meant to replace, bypass or modify any of the accepted MR safety procedures for safeguarding health care workers, patients and volunteers. 


\section{References:}

1. Legge A (2009) A review of the top 10 health technology hazards and how to minimise their risks. Nurs Times 105:17-19.

2. $\quad$ ECRI (2008) Top 10 health technology hazards. Health Devices 37:343-350.

3. Robitaille PM, Abduljalil AM, Kangarlu A et. al. (1998) Human magnetic resonance imaging at $8 \mathrm{~T}$. NMR Biomed 11:263-265.

4. Vaughan T, DelaBarre L, Snyder C et. al. (2006) 9.4T human MRI: preliminary results. Magn Reson Med 56:1274-1282.

5. Barth M, Meyer H, Kannengiesser SA et. al. (2010) T2-weighted 3D fMRI using S2-SSFP at 7 tesla. Magn Reson Med 63:1015-1020.

6. Tallantyre EC, Morgan PS, Dixon JE et. al. (2009) A comparison of 3T and 7T in the detection of small parenchymal veins within MS lesions. Invest Radiol 44:491-494.

7. Vaughan JT, Snyder CJ, DelaBarre LJ et. al. (2009) Whole-body imaging at 7T: preliminary results. Magn Reson Med 61:244-248.

8. Umutlu L, Maderwald S, Kraff O et. al. (2010) Dynamic contrast-enhanced breast MRI at 7 Tesla utilizing a single-loop coil: a feasibility trial. Acad Radiol 17:1050-1056.

9. Umutlu L, Orzada S, Kinner S et. al. (2011) Renal imaging at 7 Tesla: preliminary results. Eur Radiol 21:841-849.

10. van Elderen SG, Versluis MJ, Westenberg JJ et. al. (2010) Right coronary MR angiography at 7 T: a direct quantitative and qualitative comparison with $3 \mathrm{~T}$ in young healthy volunteers. Radiology 257:254-259.

11. Niendorf T, Sodickson DK, Krombach GA et. al. (2010) Toward cardiovascular MRI at 7 T: clinical needs, technical solutions and research promises. Eur Radiol 20:2806-2816.

12. von Knobelsdorff-Brenkenhoff F, Frauenrath T, Prothmann M et. al. (2010) Cardiac chamber quantification using magnetic resonance imaging at 7 Tesla-a pilot study. Eur Radiol 20:2844-2852.

13. Frauenrath T, Hezel F, Renz W et. al. (2010) Acoustic cardiac triggering: a practical solution for synchronization and gating of cardiovascular magnetic resonance at 7 Tesla. J Cardiovasc Magn Reson 12:67.

14. Dieringer M, Renz W, Lindel T et. al. (2011) Design and Application of a Four-Channel Transmit/Receive Surface Coil for Functional Cardiac Imaging at 7T. J Magn Reson Imaging epub ahaead of print.

15. In: U.S. Food and Drug Administration. Center for Devices and Radiological Health. (2010) MAUDE data base reports of adverse events involving medical devices. available via http://www.accessdata.fda.gov/scripts/cdrh/cfdocs/cfMAUDE/search.CFM. accessed November 15, 2010.

16. In: Auntminnie Web site. (2009) Rise in MRI accidents highlights need for magnet safety. available via http://www.auntminnie.com/index.asp?sec=ser\&sub=def\&pag=dis\&ItemID=86898. $\quad$ accessed November 15, 2010.

17. In: image the source for radiology professionals. (2009) Risky Business: MRI accidents increase fourfold in as many years. available via http://www.rtimage.com/Risky_Business_MRI_accidents_increase_fourfold_in_as_many_years/content=AFE5FAD F-B722-6933-86DFDFDB897D4A7C. accessed November 15, 2010.

18. McNeil DG. M.R.I.'s Strong Magnets Cited in Accidents. New York Times 2005 19.08.2005.

19. Administration FaD. MAUDE data base reports of adverse events involving medical devices. 2010.

20. Chen D. Boy, 6, dies of skull injury during MRI; oxygen tank becomes fatal missile in hospital. New York Times 2001 31.06.2010;B1, B5.

21. Colletti PM (2004) Size "H" oxygen cylinder: accidental MR projectile at 1.5 Tesla. J Magn Reson Imaging 19:141-143.

22. Chaljub G, Kramer LA, Johnson RF, 3rd et. al. (2001) Projectile cylinder accidents resulting from the presence of ferromagnetic nitrous oxide or oxygen tanks in the MR suite. AJR Am J Roentgenol 177:27-30.

23. Kanal E, Barkovich AJ, Bell C et. al. (2007) ACR guidance document for safe MR practices: 2007. AJR Am J Roentgenol 188:1447-1474.

24. In: U.S. Food and Drug Administration. (2009) A Primer on Medical Device Interactions with Magnetic Resonance Imaging Systems. available via http://www.fda.gov/MedicalDevices/DeviceRegulationandGuidance/GuidanceDocuments/ucm107721. htm. accessed November 15, 2010. 
25. Shellock FG, Crues JV (2004) MR procedures: biologic effects, safety, and patient care. Radiology 232:635-652.

26. Shellock FG, Woods TO, Crues JV, 3rd (2009) MR labeling information for implants and devices: explanation of terminology. Radiology 253:26-30.

27. Molyneaux DA, Ceisla J, Tsalikis D; (2009) Koninklijke Philips Electronics N.V. (BA Eindhoven, NL) assignee. Method and Apparatus for Ferrous Object and/or Magnetic Field Detection for MRI Safety United States Patent Application 20090266887

28. In: Kopp Development Inc. (2010) GaussAlert. available via http://www.koppdevelopment.com/gaussalert.html. accessed January 8th, 2010. 


\section{Figure Captions:}

\section{Figure 1:}

Basic diagram of the proposed magneto alert sensor (a). The principal circuit contains three main components: A battery, reed contacts and a signal unit. The current implementation of MALSE utilizes 7 reed contacts set at evenly spaced angles in 3 dimensions. This diagram (b) shows the 7 orientations the reed contacts are in from the points of view of 2 different planes. When combined, they offer a formation of 7 reed contacts all converging on one point, which is attached to the circuit. The outward pointing ends of the reed contacts are also attached to the circuit. When a magnetic field causes one of the reed contacts to close, the circuit will be completed and the alarm will sound. The circuit does not consume any power and the battery life time is only limited by self-discharge.

\section{Figure 2:}

Synopsis of the frequency of reed contacts of the 10-30 AT type (blue) and the 5-15 AT type (black) being activated in a given quarter gauss interval. The portfolio of reed contacts included in this study showed activation at magnetic field strengths ranging from 7-16.5 G.

\section{Figure 3:}

Scatter plot of the magnetic field strength at activation (X axis) vs. the change in field strength from activation to deactivation ( $\mathrm{Y}$ axis) for the 10-30 AT reed contacts (Black) and the 515 AT reed contacts (Orange). The average change in magnetic field strength is greater for the 5-15 AT reed contacts than for the 10-30 AT reed contacts.

\section{Figure 4:}


Picture photographs of an early MALSE prototype (a) made from standard electronic components together with a MALSE implementation (b) used for examining the MALSEs efficacy in clinical MR environments.

\section{Figure 5:}

right) Schematics of the 10 Gauss locations where the practical operation of the MALSE device in the fringe field of a $1.5 \mathrm{~T}$ (top), $3.0 \mathrm{~T}$ (center) and $7.0 \mathrm{~T}$ (bottom) MR system respectively, was photographed. The position at which MALSE got activated is marked in red while the camera position is marked in blue. The $10 \mathrm{G}, 5 \mathrm{G}$ and $1 \mathrm{G}$ lines are marked in green, red, black , starting from the magnet's iso-center.. left) Photographs of the practical operation of the MALSE device using a visual signal and an acoustic alert signal in said locations. 
a)

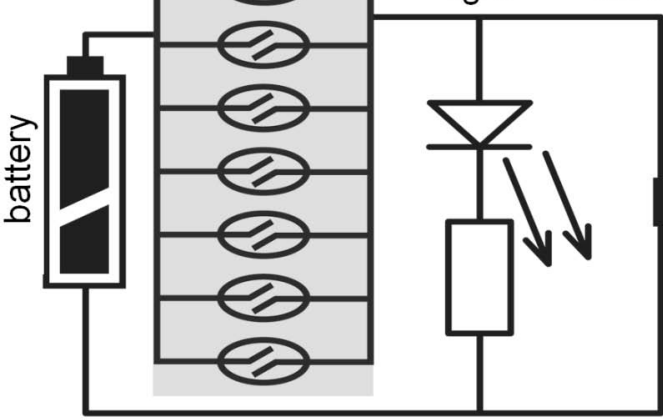

7 reed contacts light buzzer

b)
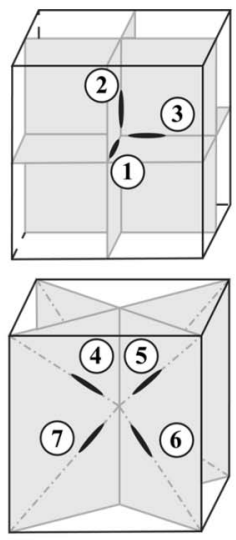


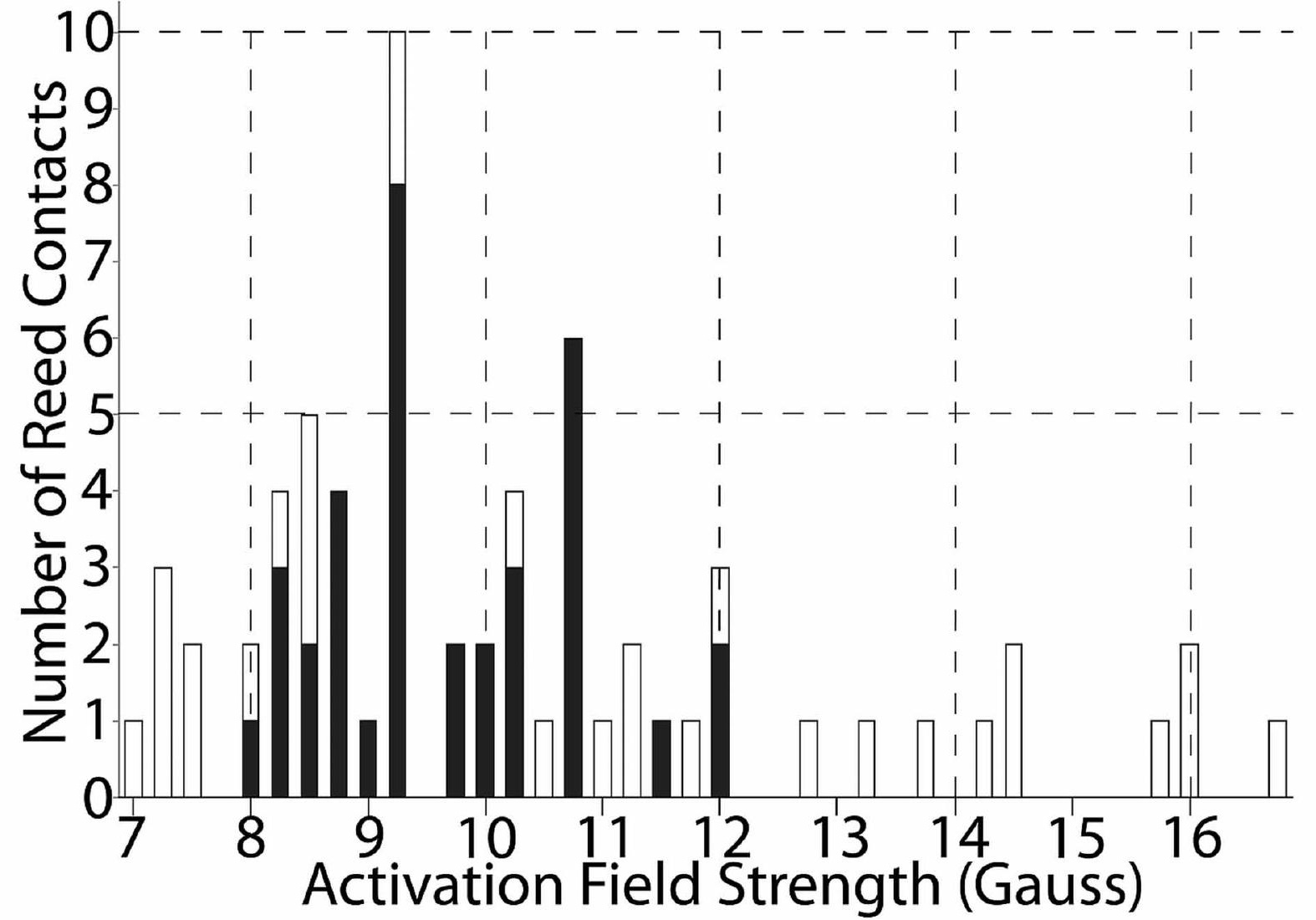




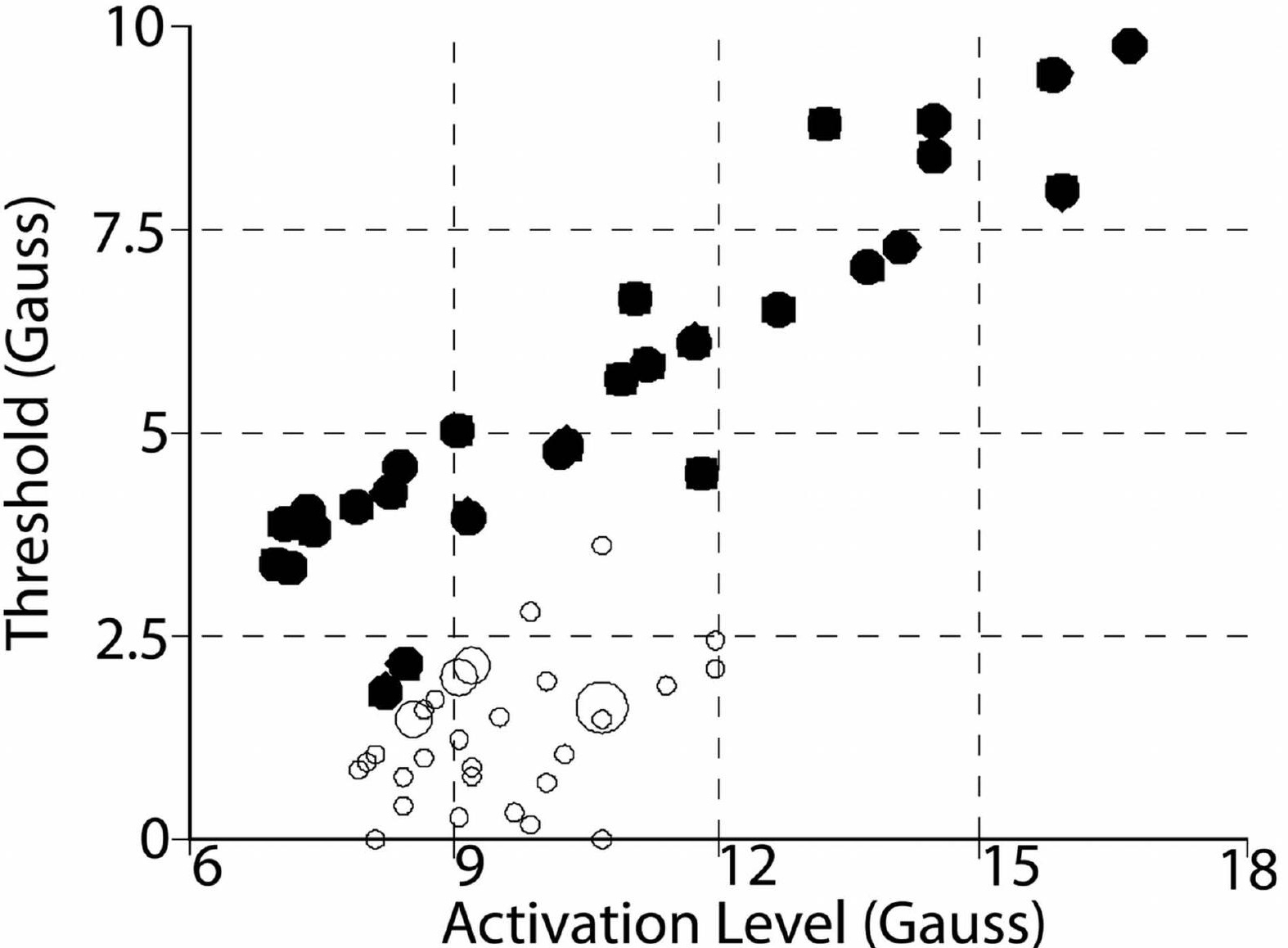



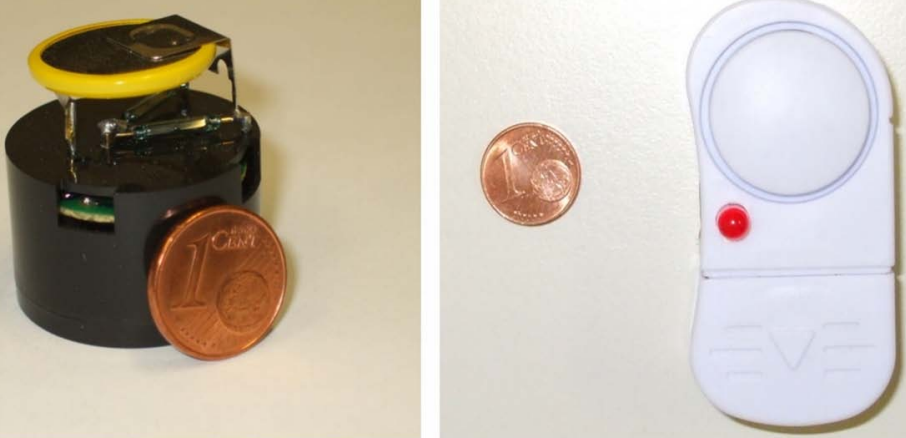


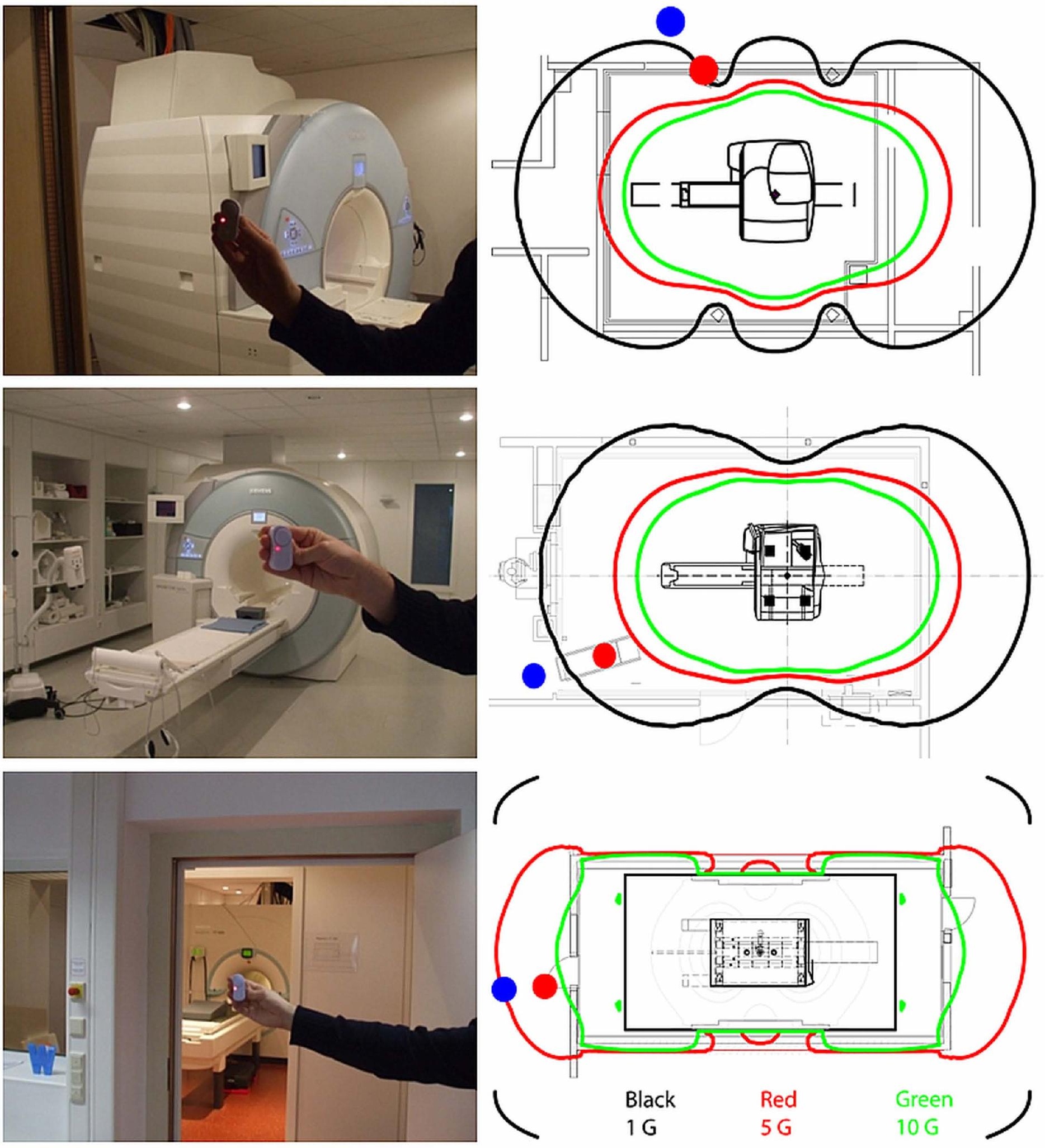

